\title{
Angiotensinogen Gene and Hypertension in Chinese
}

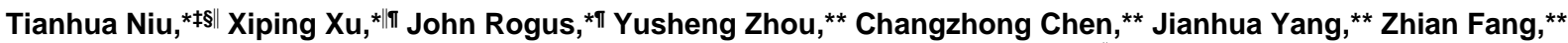 \\ Christian Schmitz, ${ }^{\ddagger}$ Jinghua Zhao, $"$ V. Srinivas Rao, ${ }^{\ddagger}$ and Klaus Lindpaintner ${ }^{\star} \neq \S \mid \neq \neq$ \\ *Program for Population Genetics, Harvard School of Public Health, Boston, Massachusetts 02115; ${ }^{\ddagger}$ Cardiovascular Division, \\ Department of Medicine, Brigham and Women's Hospital, Harvard Medical School, Boston, Massachusetts 02115; ${ }^{\S}$ Department of \\ Cardiology, The Children's Hospital, Harvard Medical School, Boston, Massachusetts 02115; "Division of Biological Sciences, Harvard \\ School of Public Health, Boston, Massachusetts 02115; "The Channing Laboratory, Department of Medicine, Brigham and Women's \\ Hospital, Harvard Medical School, Boston, Massachusetts 02115; **Anhui Meizhong Research Institute for Biomedical Sciences and \\ Environmental Health, Anhui, China; and ${ }^{\ddagger}$ Max Delbrück Centre for Molecular Medicine, Berlin, Germany
}

\begin{abstract}
The renin-angiotensin system plays a major role in regulating blood pressure and maintaining electrolyte and volume homeostasis. Previously, the angiotensinogen gene, which encodes the key substrate for renin within this system, has been reported linked to and associated with essential hypertension in White Europeans, African-Caribbeans, and Japanese. Therefore, we investigated whether the angiotensinogen gene might be similarly implicated in the pathogenesis of essential hypertension in Chinese by carrying out linkage analysis in $\mathbf{3 1 0}$ hypertensive sibling pairs. Genotypes for two diallelic DNA polymorphisms observed at amino acid residues 174 (T174M) and 235 (M235T) within the coding sequence and for two highly informative dinucleotide (GT)-repeat sequences (one in the $3^{\prime}$ flanking region, and one at a distance of $6.1 \mathrm{cM}$ from the gene) were determined. Affected sibpair analysis conducted according to three different algorithms (S.A.G.E./SIBPAL, MAPMAKER/ SIBS, and APM methods) revealed no evidence for linkage of the angiotensinogen gene to hypertension. Our data indicate that molecular variants of this gene do not appear to contribute materially to the pathogenesis of primary hypertension among Chinese (a notion supported by concomitant, direct estimates of power), and that the disease relevance of this gene may vary therefore depending on ethnicity. (J. Clin. Invest. 1998. 101:188-194.) Key words: hypertension • genetics • angiotensinogen $\cdot$ sibpair studies • linkage analysis
\end{abstract}

\footnotetext{
Klaus Lindpaintner and Xiping Xu contributed equally to this paper. Address correspondence to Klaus Lindpaintner, M.D., Cardiovascular Division, Department of Medicine, Brigham and Women's Hospital, Harvard Medical School, 75 Francis Street, Boston, MA 02115-6195. Phone: 617-732-8173; FAX: 617-264-6830; E-mail: kl@ calvin.bwh.harvard.edu or Xiping Xu, M.D., Ph.D., Program for Population Genetics, Harvard School of Public Health, 665 Huntington Avenue, Boston, MA 02115.

Received for publication 31 December 1996 and accepted in revised form 4 November 1997.
}

J. Clin. Invest.

(C) The American Society for Clinical Investigation, Inc. 0021-9738/98/01/0188/07 \$2.00

Volume 101, Number 1, January 1998, 188-194

http://www.jci.org

\section{Introduction}

Essential hypertension is a complex trait of major public health importance based on its prevalence and its association with morbidity from coronary heart disease, stroke, renal disease, peripheral vascular disease, and other disorders (1). Essential hypertension is regarded as a multifactorial condition whose onset and severity are influenced by both genetic and environmental factors. The role of genetic factors in the etiology of hypertension is supported by cross-sectional studies that document familial aggregation of the disorder, as well as by twin and adoption studies that indicate a greater degree of trait concordance among identical as compared with dizygotic twins $(2,3)$, and among natural as compared with adoptive siblings (4), respectively. While rare syndromes showing simple Mendelian inheritance (5) have been reported, the large majority of cases appears to exhibit complex polygenic patterns of inheritance where the level of blood pressure represents a remote phenotype that is governed by the combined effects of blood pressure-raising and -lowering genes (and their respective molecular variants) as well as their interaction with each other and with environmental variables.

The renin-angiotensin system contributes importantly to the regulation of vascular tone, blood pressure, cardiovascular remodeling, and electrolyte and volume homeostasis (6), and is a logical source of candidate genes for hypertension. Recent reports have implicated the gene encoding angiotensinogen $(A G T)$, the precursor of the vasoactive peptide, angiotensin II, as being genetically linked to essential hypertension in populations of Caucasian ancestry $(7,8)$ as well as in African-Caribbeans (9); in addition, an association between hypertension and the molecular variants at positions 235 and 174 of the gene has been reported by some $(7,10)$, but not by others $(8,9,11)$.

Investigations into the genetic underpinnings of complex disease rely on the ascertainment, precise phenotypic characterization, and stringent selection of large numbers of families for genetic linkage analyses. Among disease-affected sibpairs the degree of allele sharing will exceed chance only when the respective locus is causally related to the disease or when the alleles in question are in strong linkage disequilibrium with disease locus alleles. We studied the role of the $A G T$ gene and of its previously described mutations in a large, well-characterized, and highly selected cohort of hypertensive sibpairs from Anqing, a remote area in Anhui province, China. Limited migration of the population sampled as well as shared exposure to common environmental variables provide an excellent opportunity for the study of genetic influences on hypertension in this cohort. Because of the complex nature of hypertension, and because of the possibility that certain characteristics may 
serve as intermediate phenotypes that would allow stratification into pathogenetically distinct subgroups, we examined subgroups as well as the entire study sample for a possible linkage between $A G T$ and essential hypertension.

\section{Methods}

\section{Study sample}

The study was conducted in the Zongyang and Huaining counties of Anhui province, a remote, rural area of central China that offered several important advantages, including limited population mobility, low divorce rate, large average family size, and low prevalence of treated hypertension.

In 1994, 310 hypertensive sibpairs (223 sibpairs, 21 sets of 3 , and 4 sets of 4 affected siblings) were selected from the study area based on data obtained in a previously conducted survey on 20,216 individuals from the area (12). Individuals were selected if their systolic blood pressure was $\geq 140 \mathrm{mmHg}$ or if their diastolic blood pressure was $\geq 90 \mathrm{mmHg}$ regardless of treatment status. Cross-referencing of medical records disclosed that $98 \%$ of the selected subjects had a previous history of physician-diagnosed hypertension, including information about age of onset. In addition, 15 individuals who had a history of hypertension and who were on antihypertensive therapy but who had systolic blood pressure $<140 \mathrm{mmHg}$ and diastolic $<90 \mathrm{mmHg}$ were enrolled. Only subjects over the age of $15 \mathrm{yr}$, and only those without evidence for secondary forms of hypertension such as diabetes, hyperthyroidism, or glomerular nephritis were eligible. After obtaining informed consent from all individuals enrolled, a questionnaire was administered, and a screening exam performed as described previously (12).

\section{Blood pressure measurement}

Blood pressure measurements were obtained after subjects had voided, had not smoked for $15 \mathrm{~min}$, and had rested, comfortably seated, for $10 \mathrm{~min}$. Measurements were taken by arm plethysmography, using a mercury-gravity manometer with appropriately sized cuffs, by trained nurses. Triplicate measurements were taken on the same arm, waiting at least $30 \mathrm{~s}$ between readings. Systolic blood pressure was defined as Korotkoff phase I (appearance of sound), and diastolic pressure as Korotkoff phase V (disappearance of sound), respectively. The radial pulse rate was measured for $60 \mathrm{~s}$ between blood pressure determinations. Three measurements were taken and the average was used in the data analysis.

\section{Phlebotomy}

Forearm venous blood samples were then collected from each subject by venipuncture into 5-ml vacutainer tubes containing EDTA (two tubes) and citrate (two tubes). Tubes were kept on ice and were spun subsequently for $10 \mathrm{~min}$ in a refrigerated centrifuge at 2,000 $\mathrm{g}$. Plasma was subsequently removed from the cell pellet by pipetting. All samples were frozen at $-85^{\circ} \mathrm{C}$.

\section{DNA extraction}

High molecular weight DNA (200-400 kb) was extracted using adsorption to a silica matrix after lysis with a proprietary reagent (QIAGEN Inc., Chatsworth, CA), high-salt washes, and elution in $\mathrm{H}_{2} \mathrm{O}$, as described previously $(13,14)$.

\section{Genotype analyses}

Diallelic polymorphisms. The M235T and T174M polymorphisms (7) (Fig. 1) were investigated by PCR amplification of genomic DNA followed by restriction-endonuclease digestion. The variant M235T was analyzed using a modification of the method described previously (15). In brief, genomic DNA (20 ng) was amplified in a reaction containing $200 \mathrm{nM}$ of each primer (for primer sequences please refer to Table I), $50 \mathrm{mM} \mathrm{KCl}, 1.5 \mathrm{mM} \mathrm{MgCl}, 10 \mathrm{mM}$ TRIS- $\mathrm{HCl}$ (pH 9.0 at $\left.25^{\circ} \mathrm{C}\right), 0.1 \%$ Triton $\mathrm{X}-100,200 \mathrm{nM}$ of each deoxynucleotide triphos- phate, and $0.15 \mathrm{U}$ of Taq polymerase (Promega Corp., Madison, WI) in a volume of $15 \mu \mathrm{l}$. An initial denaturation for $3 \mathrm{~min}$ at $94^{\circ} \mathrm{C}$, was followed by 38 cycles of $15 \mathrm{~s}$ at $94^{\circ} \mathrm{C}, 45 \mathrm{~s}$ at $60^{\circ} \mathrm{C}$, and $45 \mathrm{~s}$ at $72^{\circ} \mathrm{C}$. The specific mismatches incorporated into the antisense primer create a Tth111I site if the $235 T$ variant is present; subsequent digestion with this enzyme at $65^{\circ} \mathrm{C}$ thus results in diagnostic fragments that are visualized by ethidium bromide staining and ultraviolet transillumination after electrophoresis on a horizontal submarine 3.5\% agarose gel. The $T 174 M$ polymorphism was genotyped as an NcoI PCRRFLP, using an analogous protocol, except for an annealing temperature of $64^{\circ} \mathrm{C}$.

Microsatellite polymorphisms. Genotyping for the $A G T$ and HLX1 (GenBank No. L21930) GT-repeats (see Fig. 1) was carried out as described previously $(7,16)$ with primer sequences shown in Table I. The $H L X 1 G T$-repeat marker has been mapped at a distance of $6.1 \mathrm{cM}$ (Kosambi) from the $A G T$ and was chosen for its high polymorphism information content (PIC) ${ }^{1}$ to provide haplotype information at the locus. For each marker, one primer was labeled at the $5^{\prime}$ end with $\left[\gamma^{-32} \mathrm{P}\right] \mathrm{ATP}$ as described previously $(7,16)$, and PCR was carried out at annealing temperatures of $66^{\circ} \mathrm{C}(H L X 1)$ and $60^{\circ} \mathrm{C}$ $(A G T)$ for 39 cycles. Reaction products were resolved over denaturing sequencing gels containing $6 \%$ polyacrylamide, $8 \mathrm{M}$ urea, and $30 \%$ formamide, and visualized by autoradiography. Several repetitive loadings per lane and run were done to improve efficacy and output. Scoring was carried out by two independent observers as described previously (17).

\section{Statistical analysis}

General. A multianalytical approach was used for the affected sibpair analysis, to provide comparability to previous investigations.

S.A.G.E./SIBPAL method. We used the S.A.G.E./SIBPAL program (18) to carry out a qualitative trait linkage analysis of $M 235 T$, T174M, AGT-GT, and HLX1-GT. This nonparametric method requires no a priori assumptions of patterns of inheritance. All subjects included in our study are sibpairs concordant for hypertension. The basic assumptions of the program are that the sibpairs of the study are selected from a random mating population and that the population is in gametic equilibrium. For each marker locus, the program uses sibling genotype data and marker allele frequencies to estimate $\overline{\hat{\pi}}$, the mean proportion of marker alleles shared identical by descent (i.b.d.) by the $n$ sibpairs and the corresponding standard deviation, $S \hat{\pi}$.

If the statistic

$\sqrt{n}\left(\frac{\bar{\pi}-.5}{S_{\text {昏 }}}\right)>1.96$

then the proportion of alleles shared i.b.d. exceeds 1:2, the level expected under the null hypothesis, and the data support the presence of linkage (at the level of $\alpha=0.05$ ). Since we only have four sets of four affected siblings out of 248 sibships (1.6\%), weighting by family size was considered unnecessary.

MAPMAKER/SIBS method. A multipoint linkage analysis was implemented using MAPMAKER/SIBS (19). In MAPMAKER/SIBS, allele sharing is determined at incremental locations along a chromosome, taking into account the genotypes of all available markers on that chromosome. Based on this allele-sharing information, a statistic (lod score) is then calculated at each chromosomal location by choosing from among all genetically feasible combinations (i.e., $z_{0}, z_{1}, z_{2}$, the probabilities that the sibs share 0,1 , or 2 alleles i.b.d., in order to maximize the likelihood. The constraints for this maximization have been shown to be $z_{2}+z_{1}+z_{0}=1, z_{2}+z_{0} \geq z_{1}, z_{1} \geq 2 z_{0}$ (20). Any regions surpassing the traditional cutoff ( $\operatorname{lod}>3.0$ ), or alternatively,

1. Abbreviations used in this paper: APM, affected pedigree method; i.b.d., identical by descent; i.b.s., identity by state; PIC, polymorphism information content. 


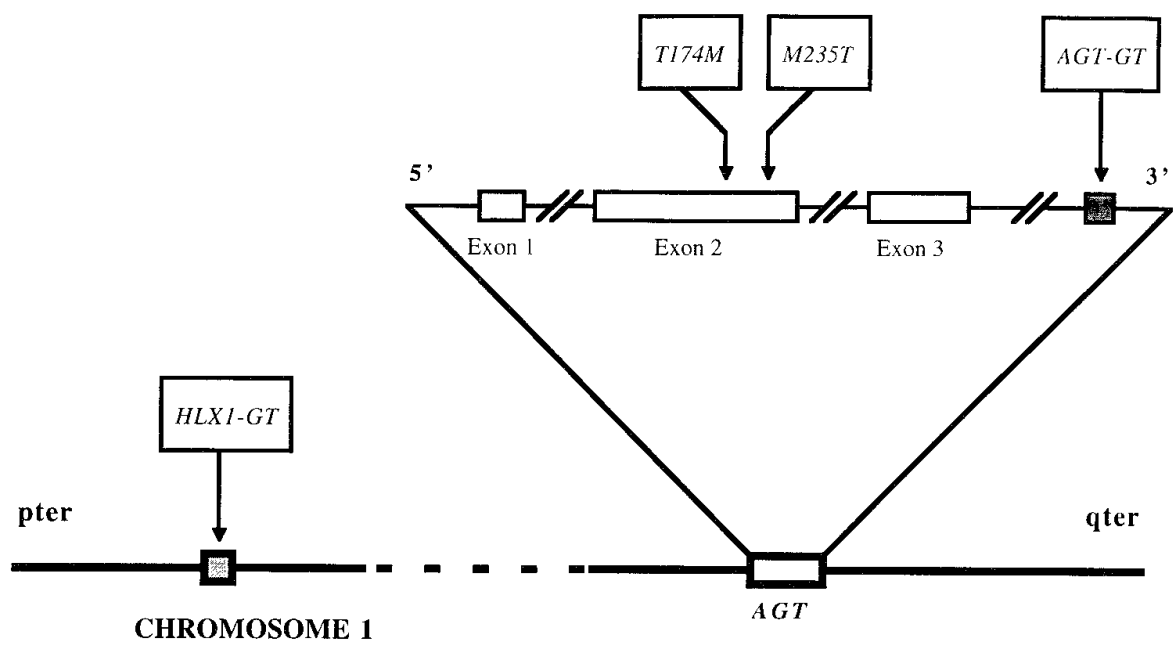

Figure 1. Schematic representation of the four polymorphic markers used in the study showing their location with respect to the angiotensinogen gene. Note that the drawing is not to scale. the cutoffs suggested by Lander and Kruglyak (21) (lod > 3.6 for significant and lod $>2.2$ for suggestive linkage), are flagged.

Affected pedigree method (APM). Using identity by state (i.b.s.), the APM method calculates a nonparametric statistic (T), which measures whether affected relatives share alleles in excess of expectation, as is the case if linkage exists $(22,23)$. Like the i.b.d. sibpair method, APM makes no explicit assumptions about mode of inheritance, penetrance, or the presence of phenocopies. To place more emphasis on the sharing of rare marker alleles between affected subjects, the pair-wise statistic, normally unweighted $\left[f_{\mathrm{A}}(p)=1\right.$, where $p$ denotes the allele frequency] can be weighted with an intermediate weight $\left[f_{\mathrm{B}}(p)=1 / \operatorname{sqrt}(p)\right]$ or with a heavy weight $\left[f_{\mathrm{C}}(p)=1 / p\right]$.

Power simulations. Assessment of the power of our sample to detect linkage was carried out by simulation as described previously by Risch (24-26), using the model which assumes first degree relatives only, and lack of parental data. Based on the specified level of recombination, on the PIC of a marker, and on a given sibling risk ratio $\left(\lambda_{\mathrm{s}}\right.$, the recurrent risk for a sibling of an affected proband proportioned on population prevalence), the probability that siblings will share 0,1 , or 2 alleles can be calculated. Using these probabilities, replicates of $\mathrm{m}$ families can be simulated, and for each replicate, the lod score calculated. The proportion of replicates exceeding the cutoff for significance (e.g., lod > 3.0) is the power to detect linkage using $\mathrm{m}$ families. To simplify calculations, markers are assumed to have equally frequent alleles. For the $A G T-G T$ (PIC $=0.86$, recombination fraction $\theta=0.00)$, a six-allele model was thus used; and for the $H L X 1-G T$ (PIC $=0.77$, recombination fraction $\theta=0.061)$, a five-allele model was used. The hypothesis being tested in our study is whether or not the $A G T$ plays a major role in the causation of essential hypertension. Hence, a single-locus model was used, and $\lambda_{\mathrm{s}}$ assumed to correspond only to the $A G T$ (i.e., $\lambda_{\mathrm{s}, A G T}$ ). The sample-specific recurrent sibling risk ratio, $\lambda_{\mathrm{s}}$, was calculated from a randomly selected group of 750 nuclear families from Anhui province in whom hypertension was defined as exceeding the $90^{\text {th }}$ percentile of the residual distribution of systolic and/or diastolic blood pressure. Power simulations were per- formed for 310, 263, and 235 affected sibpairs assuming i.b.s. (no parental genotype information available). These numbers reflect the total number of sibpairs available for the study $(n=310)$, the number of sibpairs where both members fulfilled the cutoff criteria for hypertension based on the Anhui Field Survey population data (i.e., exceeding the $90^{\text {th }}$ percentile of the residual blood pressure distribution; $n=263$ ), and the number of sibpairs where both members fulfilled the cutoff criteria for hypertension based on data from the 750 randomly selected families used to derive $\lambda_{\mathrm{s}}$ (i.e., exceeding the $90^{\text {th }}$ percentile of the residual blood pressure distribution; $n=235$ ), respectively.

\section{Results}

Among the 525 study subjects, representing 248 sibships and 310 sibpairs, there were 335 (64\%) males and 190 (36\%) females. Table II presents the clinical characteristics of the study population. Except for smoking, clinical parameters showed very small variances, supporting the contention that the study population was rather homogeneous.

The $M$ and $T$ alleles for the polymorphic marker at amino acid position 235 were present at frequencies of 0.21 and 0.79 ; the frequencies of the $T$ and $M$ alleles for the marker at position 174 were 0.93 and 0.07 . Genotype frequencies, as determined from one randomly chosen individual from each sibship, were in Hardy-Weinberg equilibrium. For the $A G T-G T$ microsatellite, 15 alleles were observed in the study population, with a PIC of 0.86 (see Table IV). The HLXI-GT marker was present in 10 allelic forms, providing a PIC of 0.77 (see Table IV).

Results of linkage analyses using the S.A.G.E./SIBPAL software (18) are presented in Table III, indicating lack of evi-

Table I. Primers Used for AGT Haplotyping Among 310 Affected Sibpairs with Essential Hypertension

\begin{tabular}{llc}
\hline \multicolumn{1}{c}{ Marker } & \multicolumn{1}{c}{$5^{\prime}$ primer sequence } & $3^{\prime}$ primer sequence \\
\hline$M 235 T$ & CCGTTTGTGCAGGGCCTGGCTCTCT & CAGGGTGCTGTCCACACTGGACCCC \\
$T 174 M$ & TGGCACCCTGGCCTCTCTCTATCT & CAGCCTGCATGAACCTGTCAATCT \\
$H L T-G T$ & GGTCAGGATAGATCTCAGCT & CACTTGCAACTCCAGGAAGACT \\
& TGCTGTCTCTGTTTCTTTCTGACC & TTCACACAAGTTCCAGCTTCCCTT \\
\hline
\end{tabular}


Table II. Clinical Characteristics of Study Sample

\begin{tabular}{|c|c|c|c|c|c|}
\hline Variable & $n$ & Mean & SD & Median & $\begin{array}{c}\text { Interquartile } \\
\text { range }\end{array}$ \\
\hline Age $(\mathrm{yr})$ & 519 & 54.7 & 10.7 & 56.0 & $48.0-62.0$ \\
\hline Age of onset of hypertension (yr) & 515 & 51.1 & 11.5 & 52.0 & $43.0-59.0$ \\
\hline $\operatorname{BMI}\left(\mathrm{kg} / \mathrm{m}^{2}\right)$ & 506 & 21.5 & 3.3 & 20.8 & $19.2-23.1$ \\
\hline $\mathrm{SBP}(\mathrm{mmHg})$ & 525 & 161.7 & 21.3 & 157.5 & $145.0-175.0$ \\
\hline $\mathrm{DBP}(\mathrm{mmHg})$ & 525 & 93.7 & 10.6 & 92.5 & $87.5-100.0$ \\
\hline Cigarette smoking (pack-yr) & 524 & 11.1 & 18.4 & 1.1 & $0.0-18.9$ \\
\hline Alcohol consumption $(\mathrm{g} / \mathrm{d})$ & 148 & 58.3 & 57.0 & 42.9 & $25.0-75.0$ \\
\hline
\end{tabular}

The number of subjects in each category is not equal to 525 due to missing values. For quantitation of alcohol consumption, only those reporting to consume were considered.

dence for excess allele sharing at any of the markers. These results were confirmed by multipoint linkage analysis using the MAPMAKER/SIBS program (19) where the probability of sharing 0,1 , or 2 alleles was estimated as $0.25,0.50$, and 0.25 (a boundary solution rendered by the program due to the constraints for $\mathrm{z}_{0}, \mathrm{z}_{1}, \mathrm{z}_{2}$ ) across the entire region spanned by the four markers, resulting in a lod score of zero. The APM (22) results, calculated either without weighting (most conservative) or using the intermediate weighting function $\left[f_{\mathrm{B}}(p)=1 /{ }_{\downarrow} p\right]$ that has been used in previous publications $(8,9)$, showed no evidence of significant linkage with any of the four markers (Table IV). We repeated the MAPMAKER/SIBS and the S.A.G.E./SIBPAL analyses on 182 sibpairs (131 sibships of 2, 11 sibships of 3 , and 3 sibships of 4 affected siblings each) in which all siblings were untreated, obtaining essentially identical results (data not shown). Similar results were obtained in S.A.G.E./SIBPAL and MAPMAKER/SIBS analyses for those 147 sibpairs in whom both siblings had blood pressure residuals (after adjustment for confounding factors) that were in the top $5^{\text {th }}$ percentile of the distribution of blood pressure residuals obtained in the general population, based on the data obtained in the previously conducted large scale survey (12) (data not shown).

Linkage studies were repeated in additional subsets of the population stratified by median age of onset of hypertension, median body mass index, and median systolic blood pressure, again failing to yield any evidence for significant linkage (data not shown).

Table $\mathrm{V}$ shows the power of our analyses as a function of $\lambda_{\mathrm{s}, A G T}$ with regard to each of the two microsatellite markers

Table III. Results of Qualitative-trait S.A.G.E./SIBPAL Analyses for T174M, M235T, AGT-GT, and HLX1-GT

\begin{tabular}{lccccc}
\hline \multicolumn{1}{c}{ Marker } & Sibpairs & $\begin{array}{c}\text { Allele sharing } \\
\text { (expected) }\end{array}$ & $\begin{array}{c}\text { Allele sharing } \\
\text { (observed) }\end{array}$ & SE & $P$ \\
\hline M235T & 309 & 0.50 & 0.51 & 0.01 & NS \\
$T 174 M$ & 308 & 0.50 & 0.50 & 0.01 & NS \\
$A G T-G T$ & 309 & 0.50 & 0.45 & 0.02 & NS \\
HLX1-GT & 302 & 0.50 & 0.48 & 0.02 & NS \\
& & & & & \\
\hline
\end{tabular}

Data calculated without weighing.
Table IV. Results of i.b.s.-based APM Analyses for M235T, T174M, AGT-GT, and HLX1-GT

\begin{tabular}{|c|c|c|c|c|c|c|}
\hline Marker & Alleles & PIC & $\begin{array}{l}\text { Nuclear } \\
\text { families }\end{array}$ & Weighting & $T$ statistic & $P$ \\
\hline \multirow[t]{3}{*}{ M235T } & 2 & 0.28 & 247 & None & 1.28 & 0.10 \\
\hline & & & & 1/sqrt(p) & 1.11 & 0.13 \\
\hline & & & & $1 / \mathrm{p}$ & 0.44 & 0.33 \\
\hline \multirow[t]{3}{*}{$T 174 M$} & 2 & 0.12 & 246 & None & -0.47 & 0.68 \\
\hline & & & & $1 /$ sqrt(p) & -1.40 & 0.92 \\
\hline & & & & $1 / \mathrm{p}$ & -1.23 & 0.89 \\
\hline \multirow[t]{3}{*}{$A G T-G T$} & 15 & 0.86 & 247 & None & -3.12 & 0.99 \\
\hline & & & & $1 /$ sqrt(p) & -2.01 & 0.98 \\
\hline & & & & $1 / \mathrm{p}$ & 0.07 & 0.47 \\
\hline \multirow[t]{3}{*}{$H L X 1-G T$} & 10 & 0.77 & 247 & None & -1.75 & 0.96 \\
\hline & & & & 1/sqrt(p) & -1.65 & 0.95 \\
\hline & & & & $1 / p$ & -0.54 & 0.70 \\
\hline
\end{tabular}

( $A G T-G T$ and HLX1-GT) based on single-locus simulations if the entire cohort of 310 sibpairs is taken into account, and if only those 263 or 235 affected sibpairs are considered that had been selected based on the criteria used to derive $\lambda_{\mathrm{s}}$ from the large scale survey data (see above).

\section{Discussion}

This study represents a large, family-based investigation into the role of $A G T$ in hypertension in Chinese. The negative results of our investigation demonstrate that in the Chinese population we studied there is no evidence that genetic variation at the angiotensinogen gene or locus contributes to a biologically relevant degree to essential hypertension. These findings, which differ from some of the results obtained previously in other ethnic groups, indicate the potential importance of ethnic origin in the assessment of genetic risk identifiers.

It is important to emphasize that the robustness and reliability of our results is enhanced by the unique opportunity we had to compare the phenotypic characteristics of our sample population with a contemporaneously established, large cross-

Table V. Power Simulations for the Two Microsatellite Markers, AGT-GT and HLX1-GT, Assuming i.b.s. Information (No Parents Available) for 310, 263, and 235 Affected Sibpairs, for a Range of Values of the Gene-specific Sibling-recurrent risk, $\lambda_{s}$ (see Methods)

\begin{tabular}{|c|c|c|c|c|c|c|}
\hline \multirow{2}{*}{$\begin{array}{l}\text { Assumed } \\
\lambda_{\mathrm{s}}\end{array}$} & \multicolumn{2}{|c|}{$\begin{array}{l}\text { Power estimate } \\
\text { (310 sibpairs) }\end{array}$} & \multicolumn{2}{|c|}{$\begin{array}{l}\text { Power estimate } \\
\text { (263 sibpairs) }\end{array}$} & \multicolumn{2}{|c|}{$\begin{array}{l}\text { Power estimate } \\
\text { (235 sibpairs) }\end{array}$} \\
\hline & $A G T-G T$ & $H L X 1-G T$ & $A G T-G T$ & $H L X 1-G T$ & $A G T-G T$ & $H L X 1-G T$ \\
\hline 1.5 & 0.34 & 0.09 & 0.25 & 0.07 & 0.19 & 0.05 \\
\hline 2.0 & 0.91 & 0.48 & 0.83 & 0.37 & 0.75 & 0.29 \\
\hline 2.5 & 1.00 & 0.77 & 0.97 & 0.64 & 0.95 & 0.55 \\
\hline 3.0 & 1.00 & 0.90 & 1.00 & 0.81 & 0.99 & 0.73 \\
\hline 3.5 & 1.00 & 0.95 & 1.00 & 0.89 & 1.00 & 0.83 \\
\hline
\end{tabular}

The $A G T$-specific $\lambda_{\mathrm{gs}}, \lambda_{A G T \mathrm{~s}}$, will represent an unknown fraction of the overall $\lambda_{\mathrm{s}}$. 
sectional database of 20,216 subjects from the same province collected only one year earlier (12). This allowed us to validate our selection of probands, and to determine a population-specific index of heritability of hypertension for direct assessment of the power of our study. The survey had revealed mean systolic and diastolic blood pressures of 114.6 and $71.2 \mathrm{mmHg}$, with standard deviations of 8.4 and $3.7 \mathrm{mmHg}$, respectively. Of note, these values are substantially lower than corresponding ones documented in Western societies (e.g., mean systolic and diastolic blood pressures of 126 and $79 \mathrm{mmHg}$ in the US [27]); therefore, even the seemingly nonstringent WHO criteria to diagnose hypertension of 140 and $90 \mathrm{mmHg}$ for systolic and diastolic blood pressure, respectively, that were used for initial selection of study subjects reflect, in actuality, a larger deviation from the norm, and thus more extreme relative blood pressure elevations than may appear at first sight, or than would have appeared if applied to a Western population. The mean residuals for systolic and diastolic blood pressure, after adjustment for age, sex, height, smoking, socioeconomic status, and exercise status (using the regression coefficients determined for these variables in the overall population) among the individuals selected as study subjects were 38.0 and 19.1 $\mathrm{mmHg}$. This places a cumulative fraction of in excess of $50 \%$ of all study subjects at or above the $95^{\text {th }}$ percentile, and of almost $80 \%$ above the $90^{\text {th }}$ percentile of the distribution of residuals determined in the population at large; and more than $90 \%$ were above the $75^{\text {th }}$ percentile (Table VI). These data confirm that our study indeed included a large majority of subjects whose blood pressure was in the extreme upper tail of the distribution of the source population.

Common complex disorders are the manifestation of a multifactorial interaction of several environmental and genetic factors. Among both, there are disease-promoting and diseaseretarding influences, and the effect of gene-environment and gene-gene interactions may modulate the effects of individual factors by affecting their magnitude, or even their direction. Thus, the effect of a given disease-contributing gene variant will vary, depending on the overall constellation of genetic and environmental factors operative in a particular individual at a particular point during development, and in a particular environment (28).

Given the difficulty of accounting for the influence of many of these confounding conditions, the (measured) magnitude of effect that a particular gene variant contributes in a complex disorder will often be modest (even if simply due to noise).

Table VI. Distribution of Subjects within Reference Population According to Systolic Blood Pressure Residuals

\begin{tabular}{lcccr}
\hline $\begin{array}{c}\text { Population } \\
\text { percentile }\end{array}$ & $\begin{array}{c}\text { Frequency } \\
\text { among sample }\end{array}$ & $\%$ & $\begin{array}{c}\text { Cumulative } \\
\text { frequency }\end{array}$ & $\begin{array}{c}\text { Cumulative } \\
\%\end{array}$ \\
\hline$<75$ th & 35 & 6.7 & 35 & 6.7 \\
75th-80th & 10 & 1.9 & 45 & 8.6 \\
81st-85th & 22 & 4.2 & 67 & 12.8 \\
86th-90th & 46 & 8.8 & 113 & 21.6 \\
91st-95th & 123 & 23.4 & 236 & 45.0 \\
$>$ 95th & 289 & 55.0 & 525 & 100.0 \\
& & & & \\
\hline
\end{tabular}

Reference population: Anhui field survey population $(n=20,216)$.
The resulting inherent lack of power is the major challenge faced by all genetic-epidemiologic investigations. It is commonly further aggravated by the difficulty of obtaining precise phenotype information (particularly in quantitative traits), by the absence of accurate data on allele frequencies (a critical issue due to the extreme sensitivity of many of the analytical algorithms to this parameter), and by lack of precise knowledge on the degree of heritability of the trait or disorder studied in the source population. To improve statistical power it is therefore critically important to optimize a number of salient features, including: (a) large sample size; $(b)$ selection of the sample from a genetically relatively homogeneous population; $(c)$ accurate documentation of and adjustment for potential confounding factors (i.e., use of regressed quantitative phenotype values); $(d)$ stringent verification of phenotype of interest; $(e)$ use of robust and redundant polymorphic markers; $(f)$ accurate, target-population-specific determination of allele frequencies; $(g)$ information on the heritability of the trait in the target population; and $(h)$ robust, redundant statistical genetic analysis. We believe that our investigation incorporates many of these features. (a) The sample size of our study (310 sibpairs) represents the largest cohort of affected sibpairs with hypertension from a single geographic area reported so far in the literature. $(b)$ Homogeneity of the population with regard to ethnicity, to socioeconomic status, and to exposure to environmental factors contributed further to noise reduction. The use of an isolated, presumably genetically more homogenous population favors the presence of fewer gene variants contributing to hypertension. (c) To account for the multiple confounding factors that might influence blood pressure in interaction with or independent of the possible contribution of $A G T$ we used the residuals of covariate-adjusted systolic blood pressure values rather than (adjusted) absolute blood pressure values, to validate subject selection and to perform calculations and analyses. $(d)$ We believe our phenotype data to be robust, based on both the analysis of our sample with respect to the covariate-adjusted population distribution, and on the selection criteria that stipulated demonstration of hypertensive blood pressure values regardless of treatment status. This was possible because, owing to limited access to continuous health care, the majority of our subjects, despite having been diagnosed previously as being hypertensive, was not receiving treatment (we verified that exclusion of the $3 \%$ of treated subjects in whom nonhypertensive blood pressure values were found did not change the overall results of the study). (e) We used two diallelic markers and two microsatellite markers, both of which are highly polymorphic. (f) We used populationspecific allele frequencies, rather than estimates from other samples. $(g)$ We had the opportunity to obtain population-specific estimates of heritability $\left(\lambda_{\mathrm{s}}\right)$, and thus to carry out meaningful power simulations. $(h)$ Lastly, we used three different statistical-genetic algorithms to analyze our data set.

To estimate the power of a genetic-epidemiological study it is necessary to know the heritability of the trait studied in the source population. While estimates of heritability for blood pressure in Caucasians have ranged from as low as $10 \%$ to as high as $60 \%$, no similar data are available for Chinese. Using a panel of 750 randomly selected nuclear families with at least two siblings (who were from the same geographic region and in whom the same set of clinical and demographic data had been collected as in our study sample) and assuming the $90^{\text {th }}$ percentile of the distribution of residuals (adjusted for blood 
pressure-relevant covariates such as age, sex, height, socioeconomic status, and smoking) as an objective cutoff for defining hypertension, we found the aggregate sibling recurrent risk ratio, $\lambda_{s}$, to be 2.4. Based on the criteria used to derive this estimate, we next carried out simulations to estimate the power rendered by our investigation, for the information content of the two microsatellite markers used, and for different assumptions of gene-specific $\lambda_{\mathrm{s}} \lambda_{\mathrm{gs}}$ (Table V). As is evident, unless one postulates that $\lambda_{\mathrm{s}, A G T}$ comprises a large proportion of overall $\lambda_{s}$, the power of the study remains rather limited. However, based on the assessments of $\lambda_{\mathrm{s}}$ and on our power simulations, we may conclude that molecular variants of $A G T$ do not account for a major proportion of the inherited (sibling-recurrent) risk of hypertension among ethnic Chinese.

We take added comfort in the finding that according to all three statistical algorithms used, and with and without weighting for rare alleles, the results of our analyses remain consistent. In previous studies results differed depending on weighting function, with significant excess allele sharing found only when weighting was used $(8,9)$. Since the APM statistic is based on i.b.s. status at the marker locus, and since this method is exquisitely sensitive to misspecification of marker allele frequencies, it is preferable to use the i.b.d.-based approaches such as S.A.G.E./SIBPAL or MAPMAKER/SIBS, to perform the affected sibpair linkage analyses. However, for reasons of comparability with previously published studies, we have also included analyses on our sample using APM.

Our study is the only affected sibpair investigation examining the possible role of $A G T$ as a contributing genetic factor to essential hypertension in a Chinese population carried out to date. Thus, our failure to reproduce earlier, positive linkage data may reflect racial/ethnic differences that are likely to exist with regard to the distribution/prevalence of individual genetic factors contributing to a complex disease. This explanation applies perhaps in particular to the sample investigated: the very characteristics that make our data robust, namely their derivation from a genetically more homogeneous population, impose certain limitations regarding their generalizability, as they will not necessarily reflect the presumably more diverse mix of hypertensinogenic gene variants present in the Chinese population at large, or in other populations of different ethnicity, such as Caucasians or African-Caribbeans (7-9).

In summary, we did not observe linkage of $A G T$ to essential hypertension in Chinese. Our results, if confirmed in additional, equally well-characterized and preferably even larger samples, demonstrate that if genetic variants of $A G T$ indeed contribute to essential hypertension, then the magnitude of this contribution is likely to be rather modest, and probably of little epidemiological relevance. In addition, the results of this study highlight the importance of accounting for ethnic background when studying the genetics of complex diseases.

\section{Acknowledgments}

We gratefully acknowledge the assistance and cooperation of the faculty and staff of the Anhui Medical University, Anqing Public Health Bureau, Yijing Hospital, Huigong Hospital, Fushan Hospital, and Haikou Hospital. We are thankful to all the participants in our study. We thank Dr. Nicholas J. Schork (Department of Biostatistics and Epidemiology, Case Western Reserve University Medical School) for insightful comments and discussions.

This work was supported in part by a Research Career Develop- ment Award (K04-HL03138-01) from the National Heart, Lung, and Blood Institute (to Dr. Lindpaintner). Some of the results of the sibpair linkage analyses were obtained by using the program package S.A.G.E., which is supported by a U.S. Public Health Service Resource Grant 1 P41 RR03655 from the Division of Research Resources.

\section{References}

1. Ward, R. 1990. Familial aggregation and genetic epidemiology of blood pressure. In Hypertension: Pathophysiology, Diagnosis, and Management. J.H. Laragh and B.M. Brenner, editors. Raven Press, New York. 81-100.

2. Levine, R.S., C.H. Hennekens, A. Perry, A. Cassady, H. Gelbrand, and M.J. Jesse. 1982. Genetic variances of blood pressure in infant twins. Am. J. Epidemiol. 116:759-764.

3. Havlik, R.J., R.J. Garrison, M. Feinleb, W.B. Kannel, W.B. Castelli, and P.M. McNamars. 1977. Blood pressure aggregation in families. Am. J. Epidemiol. 110:304-312.

4. Higgins, M.W., J.B. Keller, H.L. Metzner, F.E. Moore, and L.D. Ostrander. 1980. Studies of blood pressure in Tecumseh, Michigan. II. Antecedents in childhood of high blood pressure in young adults. Hypertension. 2 (Suppl. I):I-117-I-123.

5. Thibonnier, M., and N.J. Schork. 1995. The genetics of hypertension. Curr. Opin. Genet. Dev. 5:112-121.

6. Hall, J.E., and A.C. Guyton. 1990. Control of sodium excretion and arterial pressure by intrarenal mechanisms and the renin angiotensin system. In $\mathrm{Hy}-$ pertension: Pathophysiology, Diagnosis and Management. J.H. Laragh and B.M. Brenner, editors. Raven Press, New York. 1105-1129.

7. Jeunemaitre, X., F. Soubrier, Y.V. Kotelevtsev, R.P. Lifton, C.S. Williams, A. Charru, S.C. Hunt, P.N. Hopkins, R.R. Williams, J.-M. Lalouel, and P. Corvol. 1992. Molecular basis of human hypertension: role of angiotensinogen. Cell. 71:169-180.

8. Caulfield, M., P. Lavender, M. Farrall, P. Munroe, M. Lawson, P. Turner, and A.J.L. Clark. 1994. Linkage of the angiotensinogen gene to essential hypertension. N. Engl. J. Med. 330:1629-1633.

9. Caufield, M., P. Lavender, J. Newell-Price, M. Farrall, S. Kamdar, H. Daniel, M. Lawson, P.D. Freitas, P. Fogarty, and A.J.L. Clark. 1995. Linkage of the angiotensinogen gene locus to human essential hypertension in African Caribbeans. J. Clin. Invest. 96:687-692.

10. Hata, A., C. Namikawa, M. Sasaki, K. Sato, T. Nakamura, K. Tamura, and J.-M. Lalouel. 1994. Angiotensinogen as a risk factor for essential hypertension in Japan. J. Clin. Invest. 93:1285-1287.

11. Rotimi, C., L. Morrison, R. Cooper, C. Oyejide, E. Effiong, M. Ladipo, B.O. Osotemihen, and R. Ward. 1994. Angiotensinogen gene in human hypertension: lack of an association of the 235T allele among African Americans. Hypertension. 24:591-594.

12. Xu, X., T. Niu, D.C. Christiani, S.T. Weiss, Y. Zhou, C. Chen, J. Yang, Z. Fang, Z. Jiang, W. Liang, and F. Zhang. 1996. Environmental and occupational determinants of blood pressure in rural communities in China. Ann. Epidemiol. 7:95-106.

13. Gross-Bellard, M., P. Oudet, and P. Chambon. 1973. Isolation of highmolecular-weight DNA from mammalian cells. Eur. J. Biochem. 36:32-38.

14. Miller, S.A., D.D. Dykes, and H.F. Polesky. 1988. A simple salting out procedure for extracting DNA from human nucleated cells. Nucl. Acids Res. 16: 1215 .

15. Russ, A.P., W. Maerz, V. Ruzicka, U. Stein, and W. Gross. 1993. Rapid detection of the hypertension-associated Met235 $\rightarrow$ Thr allele of the human angiotensinogen gene. Hum. Mol. Genet. 2:609-610.

16. Sander, A., M.A. Kennedy, J.C. Rayner, and J.C. Murray. 1994. Dinucleotide repeat polymorphism for HLX1 gene. Hum. Mol. Genet. 3:219. [Published erratum appears in Hum. Mol. Genet. 3:392.]

17. Lindpaintner, K., M.A. Pfeffer, R. Kreutz, M.J. Stampfer, F. Grodstein, F. LaMotte, J. Buring, and C.H. Hennekens. 1995. A prospective evaluation of an angiotensin-converting-enzyme gene polymorphism and the risk of ischemic heart disease. N. Engl. J. Med. 332:706-711.

18. S.A.G.E., Statistical Analysis for Genetic Epidemiology, release 2.2, SIBPAL version 2.6. 1994. Computer program package available from the Department of Biometry and Genetics, Louisiana State University Medical Center, New Orleans.

19. Kruglyak, L., and E.S. Lander. 1995. Complete multipoint sib pair analysis of qualitative and quantitative traits. Am. J. Hum. Genet. 57:439-454.

20. Holmans, P. 1993. Asymptotic properties of affected sib-pair linkage analysis. Am. J. Hum. Genet. 52:362-374.

21. Lander, E.S., and L. Kruglyak. 1995. Genetic dissection of complex traits: guidelines for interpreting and reporting linkage results. Nat. Genet. 11: 241-247.

22. Weeks, D.E., and K. Lange. 1988. The affected pedigree-member method of linkage analysis. Am. J. Hum. Genet. 42:315-326.

23. Weeks, D.E., and K. Lange. 1992. A multilocus extension of the affected 
pedigree-member method of linkage analysis. Am. J. Hum. Genet. 50:859-868. 24. Risch, N. 1990. Linkage strategies for genetically complex traits. I. Multilocus models. Am. J. Hum. Genet. 46:222-228.

25 . Risch, N. 1990. Linkage strategies for genetically complex traits. II. The power of affected relative pairs. Am. J. Hum. Genet. 46:229-241.

26. Risch, N. 1990. Linkage strategies for genetically complex traits. III. The effect of marker polymorphism on analysis of affected relative pairs. Am. J.
Hum. Genet. 46:242-253.

27. Rowland, M., and J. Roberts. 1982. Blood pressure levels and hypertension in persons aged 6-74 years: United States 1976-80. In DHHS Publication No. 82-1250. National Center for Health Statistics, Hyattsville, MD.

28. Schork, N.J., S.P. Nath, K. Lindpaintner, and H. Jacob. 1996. Extensions to quantitative trait locus mapping in experimental organisms. Hypertension. 28:1104-1111. 